Rev. Psicol. (Arequipa. Univ. Catól. San Pablo) / Año 2021 / Vol 11 / Nº 2 / pp. 55-67

ISSN 2306-0565 versión impresa / ISSN 2311-7397 versión on line

\title{
Síndrome de Burnout y entusiasmo por el trabajo en docentes de Educación Básica Especial y Regular en Arequipa
}

\author{
Burnout Syndrome and Work Engagement in Special \\ and Regular Basic Education Teachers in Arequipa \\ Mariana Ramírez Chávez \\ Universidad Católica San Pablo, Arequipa, Perú \\ (iD) https://orcid.org/oooo-0002-7204-8885 \\ Correspondencia: mariana.ramirez@ucsp.edu.pe
}

\section{Resumen}

Se realizó un estudio no experimental, transversal, comparativo y correlacional con los objetivos de establecer una relación, y a su vez, comparación entre el síndrome de burnout y el entusiasmo por el trabajo en una muestra de 70 docentes de Educación Básica Especial (EBE) y Educación Básica Regular (EBR), cuyas edades oscilaron entre 25 y 60 años. Para el análisis de variables se realizó una correlación entre el burnout y entusiasmo por el trabajo, utilizando el Inventario de Burnout de Maslach y el Utrecht Work Engagement Scale (UWES) respectivamente, para demostrar su relación inversa tanto en docentes de EBE como de EBR. Asimismo, se llevó a cabo una comparación de los niveles de burnout y entusiasmo entre los dos grupos de docentes. Los resultados obtenidos mostraron que el síndrome de burnout en docentes de EBE, fue un tanto mayor que en los de EBR. Sin embargo, el nivel de entusiasmo por el trabajo fue mayor en EBE. Asimismo, los constructos evaluados (burnout y entusiasmo por el trabajo) no muestran una correlación inversa significativa en la muestra evaluada.

Palabras clave: Síndrome de burnout, entusiasmo por el trabajo, Educación Básica Especial, Educación Básica Regular.

\begin{abstract}
A non-experimental, transversal and comparative study was carried out with the purpose of establishing a relation and, in turn, a comparison between burnout syndrome and engagement, in a sample of 70 teachers of special and regular education, whose ages ranged from 25 to 60 years. For the variable analysis, a correlation was made between burnout and engagement, using the Maslach Burnout Inventory and the Utrecht Work Engagement Scale (UWES) respectively,
\end{abstract}


to determine an inverse association, as well as a comparison, in both special and regular education teachers. The results indicate that burnout syndrome level in special education teachers were higher than in regular education teachers. However, engagement levels were also higher in the special education sample. Thus, the evaluated constructs (burnout and engagement) do not show a significant inverse correlation in the referred sample.

Keywords: Burnout syndrome, engagement, special education, regular education.

\section{Introducción}

Los docentes, a pesar de tener uno de los trabajos que requieren de un tiempo de preparación considerable, muestran una gran motivación realizando la labor de la enseñanza. En líneas un poco más específicas, el entusiasmo por el trabajo o también llamado engagement, refiere un estado de la mente de carácter positivo, el cual se relaciona al trabajo y se caracteriza por la dedicación, el vigor y la absorción. Es un estado afectivo y cognitivo (Schaufeli et al., citados en Schaufeli \& Bakker, 2003). Por tal razón, en el ambiente educativo, los docentes tanto de Educación Básica Especial (EBE) como de Educación Básica Regular (EBR) deben estar actualizando la información, y además, implementando herramientas que ayuden a los alumnos dentro del aula para un aprendizaje constante (Giangreco et al., 2001).

De esta manera, en muchas ocasiones, se demostró que tal entusiasmo y motivación en los docentes puede verse seriamente afectado por el estrés, la tensión y la frustración; debido a grandes cantidades de trabajo como también a obstáculos en el proceso de enseñanza- aprendizaje, ya que eso estaría relacionado con la presión laboral que sienten (Arias \& Jiménez, 2013). En ciertos casos los niveles altos de estrés, junto con otros síntomas negativos podrían ocasionar la aparición del síndrome de burnout en los docentes. Maslach, Jackson y Leiter (1996), al realizarse el Inventario de Burnout de Maslach, definen este fenómeno como "un síndrome psicológico de cansancio emocional, despersonalización y poca realización personal que puede ocurrir entre individuos que trabajan con otras personas" (p. 192). De esta manera, tienden a caracterizarlo como una alteración entre lo que la persona siente, con lo que tiene que realizar en su centro laboral o en la situación que se encuentre. Dentro de un ambiente laboral, el estrés se manifestará con reacciones fisiológicas, emocionales y además cognitivas frente a los distintos aspectos del entorno (Arias \& Jiménez, 2013).

Sumado a ello, existen diferencias entre el tipo de estrés que acarrea la enseñanza. Arias y Jiménez (2013), exponen que existen dos tipos de estrés, el positivo y el negativo o también llamado distrés; sin embargo, como se comentó líneas arriba, el estrés en un contexto laboral y especialmente en profesiones educativas, puede estar relacionado con la conducta negativa o perturbadora de los alumnos, el poco tiempo disponible, la cultura escolar inadecuada, condiciones laborales 
poco satisfactorias, entre otras. Por otra parte, también se ven diferencias según el tipo de alumnado, si es que tienen o no discapacidad. En el contexto peruano, la docencia puede estar dirigida al alumnado sin discapacidad o con alguna condición leve (Inclusión Educativa), siendo los primeros, docentes de EBR y los segundos, docentes que orientan su enseñanza a alumnos con discapacidades moderadas, graves y severas que son los docentes de EBE (Ministerio de Educación, 2013).

A nivel mundial existe un alcance limitado de investigaciones que estudien la relación entre EBE y EBR con respecto al síndrome de burnout y el entusiasmo por el trabajo. Sin embargo, considerando estas dos últimas variables, existen estudios con respecto a otras poblaciones, llegando a ser para algunos autores, constructos opuestos (Hakanen et al., 2006; Hidalgo et al., 2019; Martínez \& Salanova, 2003), aunque, deben trabajarse de manera independiente (Schaufeli \& Bakker, 2003). Los estudios en Estados Unidos sugieren que el síndrome de burnout puede ocurrir en educación especial, en mayor proporción que en educación regular (National Association of State Directors of Special Education, citado en Roach, 2009). Por otro lado, las investigaciones sobre la incidencia del síndrome de burnout hechas en docentes de educación básica regular en Europa, refieren que los recursos (aspectos físicos, sociales, organizacionales y psicológicos) dentro del trabajo se relacionaban positivamente con el entusiasmo por el trabajo y con el compromiso laboral, mientras que el burnout se relacionaba negativamente con estas variables. Esto significaría que los docentes de EBE estarían más propensos a presentar un síndrome de burnout, y en mayor medida que los docentes de EBR (Hakanen et al, 2006).

A nivel nacional, existe escasa literatura que relacione el síndrome de burnout y el entusiasmo por el trabajo en docentes de EBR; sin embargo, en algunos estudios se encuentra una asociación entre docentes de EBR y el síndrome de burnout (Arias \& Jiménez, 2013), así como con el entusiasmo por el trabajo en docentes de EBR (Flores et al., 2015). Sin embargo, según el repositorio del Acceso Libre a Información Científica para la Innovación (ALICIA), se observa la ausencia de investigaciones que muestren la relación de estas variables en docentes de EBE; más aún en muestras de docentes de EBE y EBR (Consejo Nacional de Ciencia, Tecnología e Innovación Tecnológica [CONCYTEC], 2020).

Deacuerdo con lo mencionado, el presente estudio tiene como objetivos determinar la relación, y a su vez, las diferencias existentes entre el nivel de síndrome de burnout y del entusiasmo por el trabajo en el ámbito escolar, en docentes de EBE y docentes de EBR. Posteriormente esto sería una base para idear y realizar una intervención en aquellos docentes que presenten mayores niveles de burnout o que el nivel de entusiasmo por el trabajo sea bajo; ya que, según la literatura, la actitud y la disposición del docente para con el trabajo, influye mucho en la motivación que presentan los alumnos hacia la enseñanza, o en casos de docentes de 
EBE, influye en el progreso y la respuesta a la dinámica de los alumnos dentro del aula (Beck \& Gargiulo, 2014).

\section{Método}

El presente estudio se trató de una investigación no experimental transversal, comparativa y correlacional, por tanto se adoptó un diseño de investigación asociativo (Ato et al., 2013).

\section{Participantes}

La muestra fue no probabilística de carácter intencional (Hernández et al., 2010), y estuvo compuesta por 70 docentes, dividida en dos grupos, los cuales contaron con la misma cantidad, homogeneizando las muestras para un mayor control y una comparación más efectiva (Del Río, 2013). Deeste modo, 35 docentes pertenecieron a la modalidad de Educación Básica Especial y35 a Educación Básica Regular. Se establecieron rangos de edades, de los cuales el mayor porcentaje pertenece a edades entre 46 y 55 años, correspondiendo con el $40 \%$ de la muestra total, y dentro de un rango de 25 a 60 años. El 17.1\% fueron varones ( $\mathrm{n}=$ 12) y el $82.9 \%$ mujeres $(n=58)$.

\section{Instrumentos}

Ficha de caracterización sociodemográfica. Se trata de un cuestionario cerrado, el cual incluyó variables sociodemográficas básicas como: sexo, edad y años de enseñanza.

Cuestionario de Burnout de Maslach (Maslach \& Jackson, 1981). Esteinstrumento evalúa tres aspectos: cansancio emocional, despersonalización y realización personal. Cada aspecto está medido por una escala separada: el cansancio emocional hace referencia a estar emocionalmente exhausto por el trabajo, la subescala de despersonalización mide la respuesta insensible e impersonal acerca del estudiante, y la subescala de realización personal hace referencia a la autoeficacia de la persona dentro del trabajo, así como a la realización personal dentro de éste. Considerando la validez de la prueba, se efectuó un análisis factorial confirmatorio en donde se corrobora una validez de constructo, así como una validez de contenido mediante el coeficiente $V$ de Aiken, mostrando niveles de validez y confiabilidad adecuados (Fernández, 2002).

Utrecht Work Engagement Scale (UWES) (Salanova et al., 200o). El UWES fue desarrollado por Schaufeli y Bakker (2003), y es utilizado para medir el entusiasmo por el trabajo y sus tres dimensiones: vigor, dedicación y absorción. Para el presente estudio se hizo uso de la versión de 15 ítems (Salanova et al., 200o). El vigor se caracteriza por poseer niveles altos de energía y resiliencia mental dentro del trabajo. Asimismo, la dedicación está caracterizada por un sentido de significancia, entusiasmo, inspiración, así como de orgullo y apertura a los desafíos en la vida laboral. Por último, se puede explicar la absorción como la satisfacción que tiene la persona ante su trabajo y la felicidad que siente al estar ahí (Hakanen et al., 2006). Los resultados encontrados en la investigación de Flores et al. (2015), realizada en Lima, hallaron una correlación 
positiva entre factores vigor, dedicación y absorción. Asimismo, se encontró que las cargas factoriales de los ítems distribuidos en sus factores fue adecuada ras realizar el análisis factorial exploratorio. En el caso de la confiabilidad, es óptima con excepción de la dimensión de absorción $(\alpha=.66)$, aunque algunos autores consideran este valor como aceptable (Livia \& Ortiz, 2014).

\section{Procedimiento}

Se pidió autorización a una institución pública católica tratándose de la muestra de EBE, para así poder evaluar a los docentes calificados. Primeramente la muestra estaba premeditada para ser mucho más extensa, pero considerando la poca accesibilidad a docentes de EBE, se procesaron los datos de tan sólo 35 docentes de esta modalidad de enseñanza. Asimismo, se realizó la misma acción con docentes de EBR, aplicándose los cuestionarios en diferentes instituciones públicas. Se expuso detenidamente el consentimiento informado para explicar las normas de privacidad y confidencialidad del estudio. Es importante mencionar que no todos los cuestionarios se aplicaron de manera presencial. Los que faltaron ser aplicados (1o de EBE y 15 de EBR) fueron culminados vía telefónica y online debido al estado de emergencia en los meses de abril y mayo del 2020.

\section{Resultados}

Primeramente, se realizó un análisis descriptivo de frecuencias y porcentajes de cada una de las variables de estudio. Tomando en cuenta las dimensiones del Inventario de Burnout de Maslach para ambas muestras, la dimensión cansancio emocional se presenta en un nivel alto en el $25.7 \%$ de docentes de EBE, en nivel medio en el 31.4\% y en un nivel bajo en el 42.9\%. El mayor porcentaje se halló en el nivel bajo. Por otra parte, los docentes de EBR tuvieron resultados similares: el $\mathbf{2 2 . 9} \%$ presenta un nivel alto, el $\mathbf{1 7 . 1} \%$ un nivel medio y el $60 \%$ un nivel bajo; siendo el nivel bajo el que tiene mayor porcentaje. Considerando la dimensión despersonalización, el 85.7\% muestra un nivel bajo, el 11.4\% un nivel medio y tan sólo el 2.9\% un nivel alto en docentes de EBE. En cuanto a los docentes de EBR, el $80 \%$ mostró un nivel alto, y el $20 \%$ un nivel bajo. Por último, en la dimensión realización personal, el $8.6 \%$ de docentes de EBE presentan niveles bajos, el 34.3\% un nivel medio y el $57.1 \%$ un nivel alto; siendo este último el que tiene mayor porcentaje de profesores. En cuanto a los profesores de EBR, el 7.1\% de ellos presentan un nivel bajo, el $45.7 \%$ un nivel medio y el $47.1 \%$ restante mostró un nivel alto de realización personal, siendo este último el que tiene mayor porcentaje (ver Tabla 1). 
Tabla 1. Dimensiones de burnout en docentes de EBE y EBR

\begin{tabular}{|c|c|c|c|c|c|c|}
\hline & & \multicolumn{5}{|c|}{ Cansancio Emocional } \\
\hline & & & Bajo & Medio & Alto & Total \\
\hline \multirow[t]{4}{*}{ Educación } & EBE & Frecuencia & 15 & 11 & 9 & 35 \\
\hline & & $\%$ & $42.9 \%$ & $31.4 \%$ & $25.7 \%$ & $100.0 \%$ \\
\hline & EBR & Frecuencia & 21 & 6 & 8 & 35 \\
\hline & & $\%$ & $60.0 \%$ & $17.1 \%$ & $22.9 \%$ & $100.0 \%$ \\
\hline \multirow[t]{4}{*}{ Total } & & Frecuencia & 36 & 17 & 17 & 70 \\
\hline & & $\%$ & $51.4 \%$ & $24.3 \%$ & $24.3 \%$ & $100.0 \%$ \\
\hline & & \multicolumn{5}{|c|}{ Despersonalización } \\
\hline & & & Bajo & Medio & Alto & Total \\
\hline \multirow[t]{4}{*}{ Educación } & EBE & Frecuencia & 30 & 4 & 1 & 35 \\
\hline & & $\%$ & $85 \cdot 7 \%$ & $11.4 \%$ & $2.9 \%$ & $100.0 \%$ \\
\hline & EBR & Frecuencia & 28 & o & 7 & 35 \\
\hline & & $\%$ & $80.0 \%$ & $0.0 \%$ & $20.0 \%$ & $100.0 \%$ \\
\hline \multirow[t]{4}{*}{ Total } & & Frecuencia & 58 & 4 & 8 & 70 \\
\hline & & $\%$ & $82.9 \%$ & $5.7 \%$ & $11.4 \%$ & $100.0 \%$ \\
\hline & & \multicolumn{5}{|c|}{ Realización Personal } \\
\hline & & & Bajo & Medio & Alto & Total \\
\hline \multirow[t]{4}{*}{ Educación } & EBE & Frecuencia & 3 & 12 & 20 & 35 \\
\hline & & $\%$ & $8.6 \%$ & $34 \cdot 3 \%$ & $57.1 \%$ & $100.0 \%$ \\
\hline & EBR & Frecuencia & 2 & 20 & 13 & 35 \\
\hline & & $\%$ & $5.7 \%$ & $57.1 \%$ & $37.1 \%$ & $100.0 \%$ \\
\hline \multirow[t]{2}{*}{ Total } & & Frecuencia & 5 & 32 & 33 & 70 \\
\hline & & $\%$ & $7.1 \%$ & $45.7 \%$ & $47.1 \%$ & $100.0 \%$ \\
\hline
\end{tabular}

En referencia a las dimensiones de entusiasmo por el trabajo en docentes de EBE y EBR, se observa que, en la dimensión vigor el $74.3 \%$ de los docentes presenta un nivel muy alto, el $22.9 \%$ un nivel alto y el $2.9 \%$ restantes un nivel medio. Referentea los docentes de EBR el $62.9 \%$ presenta un nivel muy alto, el $31.4 \%$ un nivel alto y el $5.7 \%$ del total, un nivel medio. La dimensión dedicación, comprende al $71.4 \%$ de profesores de EBE que presentan un nivel muy alto, y al $28.6 \%$ de los que se ubican en un nivel alto. Los docentes de EBR muestran niveles muy altos en un $68.6 \%$, el $28.6 \%$ presenta un nivel altoy el $2.9 \%$ un nivel medio. Considerando la dimensión absorción, el 71.4\% de docentes de EBE presentan un nivel muy alto, mientras que el $22.9 \%$ muestran niveles altos y el $2.9 \%$ un nivel medio y bajo. Los docentes de EBR presentan niveles muy altos en un $34.3 \%$, niveles altos en un $45.7 \%$, el $14.3 \%$ presentó un nivel medio y $2.9 \%$ en niveles bajos y muy bajos (ver Tablaz). 
Tabla 2. Dimensiones de entusiasmo por el trabajo en docentes de EBE y EBR

\begin{tabular}{|c|c|c|c|c|c|c|c|c|}
\hline & & & & & Vigc & & & \\
\hline & & & Muy bajo & Bajo & Medio & Alto & Muy alto & Total \\
\hline & FBF & Frecuencia & o & o & 1 & 8 & 26 & 35 \\
\hline Fducación & EBE & $\%$ & $0.0 \%$ & $0.0 \%$ & $2.9 \%$ & $22.9 \%$ & $74 \cdot 3 \%$ & $100.0 \%$ \\
\hline Luucderion & FBR & Frecuencia & o & o & 2 & 11 & 22 & 35 \\
\hline & LDK & $\%$ & $0.0 \%$ & $0.0 \%$ & $5.7 \%$ & $31.4 \%$ & $62.9 \%$ & $100.0 \%$ \\
\hline Total & & Frecuencia & o & o & 3 & 19 & 48 & 70 \\
\hline forat & & $\%$ & $0.0 \%$ & $0.0 \%$ & $4.3 \%$ & $27.1 \%$ & $68.6 \%$ & $100.0 \%$ \\
\hline & & & & & Dedica & & & \\
\hline & & & Muy bajo & Bajo & Medio & Alto & Muy alto & Total \\
\hline & FBF & Frecuencia & o & o & o & 10 & 25 & 35 \\
\hline Fducación & LDL & $\%$ & $0.0 \%$ & $0.0 \%$ & $0.0 \%$ & $28.6 \%$ & $71.4 \%$ & $100.0 \%$ \\
\hline & FRP & Frecuencia & o & o & 1 & 10 & 24 & 35 \\
\hline & LDK & $\%$ & $0.0 \%$ & $0.0 \%$ & $2.9 \%$ & $28.6 \%$ & $68.6 \%$ & $100.0 \%$ \\
\hline Tats & & Frecuencia & o & o & 1 & 20 & 49 & 70 \\
\hline 10 tal & & $\%$ & $0.0 \%$ & $0.0 \%$ & $1.4 \%$ & $28.6 \%$ & $70.0 \%$ & $100.0 \%$ \\
\hline & & & & & Absorc & & & \\
\hline & & & Muy bajo & Bajo & Medio & Alto & Muy alto & Total \\
\hline & FBF & Frecuencia & o & 1 & 1 & 8 & 25 & 35 \\
\hline Fducación & LDL & $\%$ & $0.0 \%$ & $2.9 \%$ & $2.9 \%$ & $22.9 \%$ & $71.4 \%$ & $100.0 \%$ \\
\hline Lutedeton & FBR & Frecuencia & 1 & 1 & 5 & 16 & 12 & 35 \\
\hline & & $\%$ & $2.9 \%$ & $2.9 \%$ & $14.3 \%$ & $45 \cdot 7 \%$ & $34.3 \%$ & $100.0 \%$ \\
\hline Total & & Frecuencia & 1 & 2 & 6 & 24 & 37 & 70 \\
\hline & & $\%$ & $1.4 \%$ & $2.9 \%$ & $8.6 \%$ & $34.3 \%$ & $52.9 \%$ & $100.0 \%$ \\
\hline
\end{tabular}

Por otra parte, tras hacer un análisis de el síndrome de burnout y el entusiasmo correlación mediante la prueba Rho de por el trabajo en los docentes de EBE y Spearman se encontró una relación nula EBR $(\mathrm{r}=.058 ; \mathrm{p}=.631)$, como se aprecia carente significación estadística entre en la Tabla 3.

Tabla 3. Relación entre síndrome de burnout y entusiasmo por el trabajo en docentes de EBE y EBR

\begin{tabular}{|llc|}
\hline & Rho de Spearman & $\begin{array}{c}\text { Entusiasmo } \\
\text { por el trabajo }\end{array}$ \\
\hline Síndrome de Burnout & $\mathrm{p}$-valor & .058 \\
& $\mathrm{~N}$ & .631 \\
& & 70 \\
\hline
\end{tabular}

Finalmente, al realizarcomparaciones entre de lavariable deentusiasmo por el trabajo, sí los docentes de EDE y EBR mediante la seencontraron diferencias estadísticamente prueba U de Mann Whitney, no se hallaron significativas $(U=440 ; Z=-2.028 ; p=.043)$, lo diferencias estadísticamentesignificativas en cual sugiereque los docentes de EBE presenla variable de burnout $(\mathrm{U}=590.5 ; \mathrm{Z}=-\mathbf{2 . 2 5 9 ;} \tan$ un mayor entusiasmo por el trabajo que $\mathrm{p}=$.796) (Tabla 4). Sin embargo, en el caso los docentes de EBR. 
Tabla 4. Comparación de síndrome de burnout y entusiasmo por el trabajo en docentes de EBE y EBR

\begin{tabular}{|lllcccc|}
\hline & Educación & N & $\begin{array}{c}\text { Rango } \\
\text { promedio }\end{array}$ & U de Mann-Whitney & $\mathbf{Z}$ & $\mathbf{p}$ \\
\hline Síndrome & EBE & 35 & 36.13 & 590.500 & -.259 & .796 \\
de Burnout & EBR & 35 & 34.87 & & & \\
& Total & 70 & & & & \\
\hline \multirow{2}{*}{$\begin{array}{l}\text { Entusiasmo } \\
\text { por el trabajo }\end{array}$} & EBE & 35 & 40.43 & 440.000 & -2.028 & .043 \\
& EBR & 35 & 30.57 & & & \\
\hline
\end{tabular}

\section{Discusión}

El síndrome de burnout manifestado en los docentes, puede expresarse como el agotamiento ocasionado por distintos factores, ya sea por la responsabilidad que el profesor tenga con sus estudiantes, el comportamiento de los mismos, el salario que recibe, entre otros factores. Asimismo, el nivel de entusiasmo por el trabajo o engagement en los docentes, también es un tema esencial, ambos constructos, aunque independientes revelan ser contrapuestos, de modo que a mayor burnout menor es el engagement, y viceversa (Hansen, 2012; Manzano, 2002; Schaufeli \& Bakker, 2003).

En general, los docentes de EBE tienden a presentar mayores puntuaciones en las dimensiones del síndrome de burnout que los docentes de EBR. Por esta razón, en el ambiente de educación especial (en el contexto español), los problemas presentados por los docentes como el estrés, ansiedad, entre otros; son mayores que en cualquier otro ámbito educativo
(Polaino-Lorente, citado en Franco, et al., 2009). En ese sentido, Hakanen et al. (2006) postulan que los docentes de EBE tienden a presentar mayores niveles de burnout que los docentes de EBR en un contexto europeo.

Sin embargo, en consideración con las dimensiones de burnout, en nuestra investigación se halló que la despersonalización es mayor en docentes de EBR que en los docentes de EBE. Probablemente esta diferencia se deba a las variables sociolaborales como los años de enseñanza que, en EBE hubo más docentes que ejercían en su puesto de 15 años a más (20 docentes de 35) y en EBR solamente hubo 10 de 35 evaluados que trabajan más de 15 años en su institución educativa; lo cual se vería apoyado por el estudio de Ruiz-Calzado y Llorent (2018) quienes encontraron en una muestra española, que los profesionales que laboran más de 21 años, en su centro educativo, tienden a padecer más burnout que aquellos que son más inexpertos. De la misma manera, tomando en cuenta las dimensiones del entusiasmo por el 
trabajo, se presentan en mayores niveles en docentes de EBE que en docentes de EBR, en cuestión de vigor, dedicación y absorción, sugiriendo que los docentes que se dedican a la enseñanza de alumnos con alguna discapacidad tienen un sentido mayor de responsabilidad para con ellos (Giangreco et al., 2001).

Consiguientemente, la relación entre el burnout y entusiasmo por el trabajo en el estudio presenta una correlación nula no significativa. Sin embargo, es importante recordar que, a pesar que estas variables en distintas ocasiones son consideradas como constructos que se relacionan inversamente (Hakanen et al., 2006; Hidalgo et al., 2019; Maslach \& Leiter, citado en Martínez \& Salanova, 2003; Salanova et al., 2000); tanto así que el engagement es considerado como el "polo opuesto" del síndrome de burnout (Martínez \& Salanova, 2003), esta relación no siempre se va a encontrar en todas las investigaciones realizadas.

Por otra parte, respecto a las diferencias entre los niveles de burnout y engagement para docentes de EBE y EBR en un contexto arequipeño, los resultados refutan la mayoría de la literatura estudiada; ya que, en cuanto a niveles de engagement, se reportó que los docentes de EBR presentan un entusiasmo mayor que los docentes de EBE (Roach, 2009). Sin embargo, los docentes de EBR pueden presentar problemas de otra índole (Giangreco et al., 2001) relacionados con las condiciones laborales y el propio trabajo docente (Arias \& Jiménez, 2013). Además, en el caso de docentes de EBE, a pesar que el estrés laboral es elevado o moderado, mantienen el compromiso con sus alumnos que muchas veces tienen discapacidades severas (Hidalgo et al., 2019).

Dentro de las limitaciones observadas en el presente trabajo, se encuentra que, en cuanto a la cantidad muestral, inicialmente el estudio se realizaría con 200 docentes (10o para cada modalidad), considerando que, según el informe, el total de la población de docentes de EBE en Arequipa es de 152 (MINEDU, 2015), y el número de docentes de EBR es mucho mayor (Instituto Nacional de Estadística e Informática [INEI], 2020). Sin embargo, para equiparar la muestra de ambas modalidades se tuvo que reducir la muestra total. A ello se suma que, en el contexto de la pandemia por la COVID-19, ha sido muy difícil contar con la colaboración de los profesores de ambas modalidades. Por otra parte, en cuanto al estado del arte, no se lograron encontrar muchos estudios e investigaciones que soporten los objetivos planteados; ya que, como se ha mencionado anteriormente, la información encontrada sobre burnout y engagement, considerando la EBE y EBR, es escasa a nivel nacional.

Dicho esto, se sugiere que, para futuros estudios se consideren muestras mucho más representativas, y que también se estudie la relación del burnout y 
el engagement en distintos niveles de enseñanza, ya que algunos estudios recientes han reportado diferencias en los niveles de burnout entre los docentes de nivel escolar y universitario (Arias et al., 2019). También, se sugiere tomar en cuenta la medición de variables sociodemográficas que puedan complementar el estudio del tema tratado, tales como el grado de instrucción, nivel de enseñanza, años de experiencia, etc. Así, se podrán observar mejor las causas o las consecuencias que las variables de estudio pueden tener en los docentes de EBE y EBR.

\section{Financiamiento}

La presente investigación fue autofinanciada.

\section{Conflictos de interés}

La autora declara que no tiene conflictos de interés.

\section{Agradecimiento}

La autora agradece el apoyo académico brindado por la Mg. Milagros Cahuana Cuentas para la realización de este estudio. 


\section{Referencias}

Arias, W. L., \& Jiménez, N. A. (2013). Síndrome de burnout en docentes de Educación Básica Regular de Arequipa. Educación, 22(42), 53-76. http://revistas.pucp.edu. pe/index.php/educacion/article/view/5291

Arias, W. L., Huamani, J. C., \& Ceballos, K. D. (2019). Síndrome de burnout en profesores de escuela y universidad: Un análisis psicométrico y comparativo en la ciudad de Arequipa. Propósitos y Representaciones, 7(3). http://dx.doi.org/10.20511. pyr2019.v7n3.390

Ato, M., López, J. J., \& Benavente, A. (2013). Un sistema de clasificación de los diseños de investigación en psicología. Anales de Psicología, 29(3), 1038-1059. https:// www.redalyc.org/pdf/167/16728244043.pdf

Beck, C. L., \& Gargiulo, R. M. (2014). Burnout in teachers of retarded and nonretarded children. The Journal of Educational Research, 76(3), 169-173. https://doi.org/10. 1080/00220671.1983.10885444

Consejo Nacional de Ciencia, Tecnología e Innovación Tecnológica (CONCYTEC) (2020). Acceso Libre a Información Científica para la Innovación [ALICIA]. CONCYTEC. https://alicia.concytec.gob.pe/vufind/

Del Río, D. (2013). Diccionario-Glosario de Metodología de la Investigación Social. Editorial UNED. https://url2.cl/xbv8p

Flores, C., Fernández, M., Juárez, A., Merino, C., \& Guimet, M. (2015). Entusiasmo por el trabajo (Engagement): Un estudio de validez en profesionales de la docencia en Lima, Perú. Liberabit, 21(2), 195-206. http://www.scielo.org.pe/scielo. php?pid=S1729-48272015000200003\&script=sci_arttext

Franco, C., Mañas, I., \& Justo, E. (2009). Reducción de los niveles de estrés, ansiedad y depresión en docentes de educación especial a través de un programa de mindfulness. Revista de Educación Inclusiva, 2(3), 11-22. https://dialnet.unirioja. es/servlet/articulo?codigo $=3082166$

Giangreco, M. F., Broer, S. M., \& Edelman, S. W. (2001). Teacher engagement with students with disabilities: Differences between paraprofessional service delivery models. The Association for Persons with Severe Handicaps, 26(2), 75-86. https:// journals.sagepub.com/doi/10.2511/rpsd.26.2.75 
Hakanen, J., Bakker, A. B., \& Schaufeli, W. B. (2006). Burnout and work engagement among teachers. Journal of School Psychology, 43, 495-513. http://www.sciencedirect.com/science/article/pii/Soo22440505000890

Hansen, D. J. (2012). Engagement y Burnout. https://url2.cl/wjMt2

Hernández, R., Fernández, C., \& Baptista, P. (2010). Metodología de la Investigación. McGraw-Hill.

Hidalgo, J. A., Acle, G., García, M., \& Tovalin, H. (2019). Burnout, resiliencia y compromiso laboral en maestros de educación especial. Educación y Ciencia, 8(51), 48-57. https://www.researchgate.net/publication/333389864

Instituto Nacional de Estadística e Informática (INEI) (2020). Estadísticas. INEI. https://www.inei.gob.pe/buscador/?tbusqueda=docentes

Manzano, G. (2002). Burnout y engagement. Relación con el desempeño, madurez profesional y tendencia al abandono de los estudiantes. International Journal of Social Psychology, 17(3), 237-249. https://doi.org/10.1174/02134740260372973

Martínez, I. M., \& Salanova, M. (2003). Niveles de Burnout y Engagement en estudiantes universitarios. Relación con el desempeño y desarrollo profesional. Revista de Educación, 330, 361-384. http://www.want.uji.es/wp-content/ uploads/2017/o3/2003_Mart\%C3\%ADnez-Salanova.pdf

Maslach, C., \& Jackson, S.E. (1981). The measurement of experienced Burnout. Journal of Occupational Behaviour, 2, 99-113. https://doi.org/10.1002/job.4030020205

Maslach, C., Jackson, S.E., \& Leiter, M. (1996). The Maslach Burnout Inventory Manual. Consulting Psychologists Press. https://www.researchgate.net/ publication/277816643_The_Maslach_Burnout_Inventory_Manual

Ministerio de Educación (2013). Diseño Curricular Nacional de Educación Básica Regular. Proceso de Articulación. Fimart S.A.C.

Ministerio de Educación (2015). Listado Padrón de Instituciones de Educación Especial (CEBE y PRITE) MINEDU.

Roach, A. (2009). Teacher Burnout: Special education versus regular education. Theses, Dissertations and Capstones, 1-22. http://mds.marshall.edu/cgi/viewcontent.cgi ?article $=1806 \&$ context $=$ etd 
Ruiz-Calzado, I., \& Llorent, V. J. (2018). El Burnout en los profesionales de la educación que trabajan con personas con discapacidad en Córdoba (España). Influencia de las variables laborales. Educación XXI, 21(2), 373-393. https://doi.org/10.5944/ educXX1.15459

Salanova, M., Schaufeli, W. B., Llorens, S., Peiró, J. M., \& Grau, R. (200o). Desde el «burnout» al «engagement»: ¿Una nueva perspectiva? Revista de Psicología del Trabajo y de las Organizaciones, 16(2), 117-134. https://journals.copmadrid.org/ jwop/files/63236.pdf

Schaufeli, W. B., \& Bakker, A. B. (2003). Utrecht work engagement scale: Preliminary Manual. Occupational Health Psychology Unit. Utrecht University. https://www. wilmarschaufeli.nl/publications/Schaufeli/Test\%2oManuals/Test_manual_ UWES_Espanol.pdf

Recibido: 29 de setiembre de 2020

Revisado: 10 de marzo de 2021

Aceptado: 6 de agosto de 2021 